\title{
Study on Scientific Research Performance Evaluation of Universities for Traditional Chinese Medicine--Take Hubei University of Traditional Chinese Medicine as an example
}

\author{
Chun-Yan LI ${ }^{1, a}$, Ting Kong ${ }^{2, b}$, Yuan Sun ${ }^{3, c_{*}}$ \\ ${ }^{1}$ School Of Marxism, Hubei University of traditional Chinese Medicine,Wuhan, China \\ ${ }^{2}$ Hubei Three Gorges Polytechnic, Yichang, China \\ ${ }^{3}$ Science and Technology Department,Hubei University of traditional Chinese Medicine,Wuhan, \\ China \\ a156738577@qq.com, bkongting87@126.com, Elyn1202@hbtcm.edu.cn
}

Keywords: Performance Evaluation, Traditional Chinese Medicine

\begin{abstract}
The paper analyzed some indexes of scientific research performance evaluation of teachers in universities for TCM by taking Hubei University of Traditional Chinese Medicine (HBTCM for short) as an example and put forward to the question how to evaluate scientifically and effectively their performance is propelling for the improvement of teachers' qualities and the realization of the strategic objectives of TCM. It is of great significance to study the performance evaluation of university teachers, especially the evaluation of scientific research performance, to establish and improve the system of teachers' performance evaluation.
\end{abstract}

\section{Introduction}

The universities for traditional Chinese medicine (TCM) are the source of inheritance and innovation of traditional knowledge of TCM, they undertake three major functions: talent cultivation, scientific research and social services. Teachers are the principal part of universities for TCM, their work enthusiasm degree is not only related to the quality of talents of their universities but also related to the realization of strategic objectives of their universities. Moreover, a wellqualified, efficient and well-structured teachers' team is a fundamental guarantee for the realization of the strategic objectives of universities for TCM. How to evaluate scientifically and effectively their performance is propelling for the improvement of teachers' qualities and the realization of the strategic objectives of TCM. Therefore, it is of great significance to study the performance evaluation of university teachers, especially the evaluation of scientific research performance, to establish and improve the system of teachers' performance evaluation.

\section{Conceptions}

\section{Performance evaluation}

In literatures, performance evaluation is presented as performance review, performance evaluation and performance appraisal and so on. As a formal individual evaluation system, performance evaluation is the most effective part of performance management. Good performance evaluation is conducive to the effective combination of organizational goals and individual goals for achieving higher organizational efficiency. Performance evaluation refers to a process, which bases the organization's strategic goals, according to the facts and working requirements, with the help of a type of structured set of index system, using scientific system of evaluation methods, to evaluate the individual in the completion of the task for a certain period of time, responsibility fulfillment degree and its own development condition, and the evaluation results feedback to individual. Generally speaking, the purpose of the performance evaluation not only lies in the rewards, but improving the 
individual performance, mobilizing individual' work initiative , improving the satisfaction of the individual with a sense of accomplishment and at the same time realizing the organization's strategic goals, finally achieving the organization and individual development of "win-win".

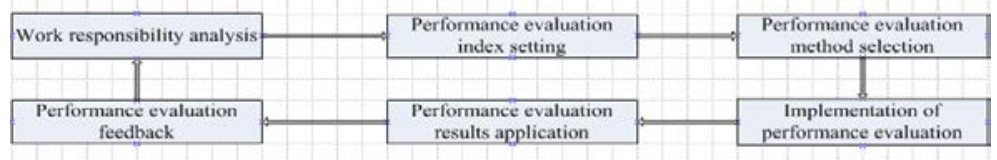

The performance evaluation process

Performance evaluation of university teachers

The evaluation of teachers' performance in universities is the reified result of our country and universities for teachers' requirements; it is one of performance evaluations. It is a comprehensive process which means under the guidance of the correct value concept, according to the objectives and tasks of universities, to formulate the corresponding evaluation system, and evaluate teachers' labor process, labor behavior and labor result, finally feedback the evaluation results to the teachers, it is a kind of guidance and specification for teachers' behavior. The performance evaluation of teachers in universities for TCM is similar.

\section{Principles \&Methods}

\section{Performance evaluation principles of university teachers for TCM}

(1) The orientation principle

It embodies the overall goal and development strategy of our country. Moreover, the performance evaluation of university teachers for TCM also reflects the expectation of the management of universities and guides the development of teachers' research work. It is helpful to regulate the scientific research behavior of university teachers for TCM and meet the needs of the country, universities and society.

(2) The objectivity principle

The performance evaluation of university teachers for TCM needs to be based on the objective facts of teachers' work to evaluate their value and cultivate teachers' sense of fairness and identity.

(3) The motivational principle

The performance evaluation should reflect not only fairness, but also the reasonable gap between teachers, so as to motivate teachers to keep learning new knowledge, improve their own abilities and realize the professional development of teachers.

(4) Competitive principle

The performance evaluation should fully mobilize the enthusiasm, initiative and creativity of teachers in universities for TCM, consequently, to improve the operation efficiency of the organization.

The performance evaluation model of teachers in universities for TCM mostly adopts quantitative evaluation, such as analytic hierarchy process and fuzzy evaluation method. The main feature of this model is the use of mathematical methods to determine the index weight of the performance evaluation, which is indexed to the teacher's work content and output, so that the evaluation criteria and evaluation results are presented in quantitative form. The performance evaluation system of scientific research mainly includes three categories: scientific research projects, achievements and awards, each category contain a number of sub items. Such as the level of scientific research projects, the number of papers, the grade of the scientific achievement award and so on.

\section{Performance evaluation indexes of scientific research in universities for TCM}

Performance evaluation of scientific research in universities for TCM refers to the judgment of teachers' scientific research work and the achievements obtained in the scientific research process in universities for TCM in accordance with certain value orientation. 
Take Hubei university of traditional Chinese medicine as an example, performance evaluation indexes of scientific research mainly include academic papers, academic works, scientific research projects, patents and scientific research achievement awards.

(1) Academic papers

Academic paper is the main form of scientific research achievement of teachers in universities for TCM. Most original innovation results of important significance are published in the form of academic papers.

The quality of academic papers is closely related to the citation, publication level, influencing factor and cited times. Therefore, the comprehensive evaluation of academic papers can be based on these indexes mentioned above: the status of the published paper, the authority of the periodical, the influencing factors of the periodical and the cited situation. The paper cited Frequency is one of the important indexes that reflect the influence of the paper.

(2) Academic works

Academic work refers to the published work completed by teachers, which is one of the contents of scientific research performance evaluation. The performance evaluation index of academic works includes the categories of works, the level of publishing houses and the words of works and so on.

(3) Scientific research projects

Research project refer to longitudinal scientific research project or various forms of horizontal scientific research project undertaken by teachers. It is an important index to measure teachers' scientific research strength and scientific research level. Scientific research project performance evaluation includes the scientific research category, project funds and the participants ranking in detail.

\section{(4) Patents}

Patent is one of the important forms of original innovation or independent research. It is an important strategic resource. This kind of index includes two aspects: the patent type and the effective transfer funds.

(5) Scientific research achievement awards

Scientific research achievement award refers to the different categories and levels of award teachers received.

It is also an important index reflecting the research and innovation ability of teachers. It includes two aspects: scientific research achievement award category and level.

The researcher takes Hubei University of Traditional Chinese Medicine (HUTCM) as an example.

(1) Basic score of academic papers

Academic papers evaluated by HUTCM include papers indexed by international five indexes (SCI, SSCI, EI, A\&HCI, CPCI), chinese academic papers (China humanities and social sciences top journals, CSSCI\&CSCD, CSSCI-extened \& CSCD-extened, CSTPCD), Chinese Publishes(People's Daily and Guangming daily theory edition, Xinhua Digest, HBDXTSG). SCI partition is based on the latest division of Chinese academy of sciences, SSCI partition is based on the latest division of Nanjing University. The following table shows basic score of papers indexed by international five indexes.

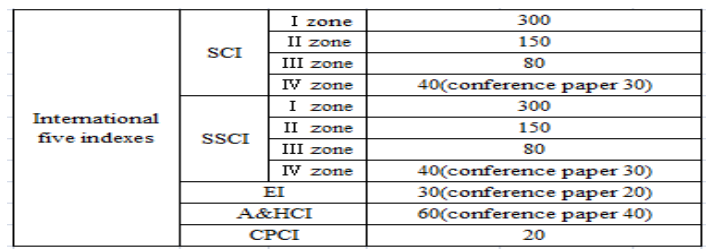

Table 1

(2) Basic score of academic works 
Basic score of academic works is calculated according to teachers' role in the works and the number of words written by teachers. The teachers' role includes editor-in-chief, associate editor, and coeditor and so on.

\begin{tabular}{|c|c|}
\hline Roles & Basic scores \\
\hline written & the number of words* $8 * 0.0001$ \\
\hline compile & the number of words $* 4 * 0.0001$ \\
\hline editor-in-chief & the number of words*2*0.1/n \\
\hline associate editor & the number of words $* * 0.01 / \mathrm{m}$ \\
\hline coeditor & the number of words*2 \\
\hline
\end{tabular}

\section{Table 2}

$n$ in the table 2 is the number of editor-in-chiefs, $m$ in the table 2 is the number of associate editors.

(3) Basic score of scientific research projects

Basic score of scientific research projects is calculated according to the actual funds to the account. The funds of natural sciences can be calculated 15 scores per ten thousand yuan, the funds of social sciences can be calculated 20 scores per ten thousand yuan.

(4) Basic score of patents

Patents evaluated cover Chinese patent and international invention patent.

Basic score of Chinese patent is 80 scores each item, Basic score of international invention patent is 200 scores each item.

(5) Basic score of scientific research achievement awards

The awards are divided into natural sciences and social sciences. Both contain national level, provincial level, Bureau level and so on. Every level is divided into different classes scoring different scores.

\section{Discussion}

Performance evaluation of scientific research of teachers in universities for TCM has achieved initial success, however, there are some problems in the process of performance evaluation. At the level of performance evaluation system, the target realization is paid more attention, the professional development of teacher is undervalued; in the models of performance evaluation, the role of organization is dominant, the participation function of teacher is light; At the level of performance evaluation index, the quantity standard is heavy, the quality requirement is light; At the level of the application of performance evaluation results, the use of evaluation results is heavy, the feedback of evaluation results is light.

\section{Suggestions}

For the sake of improving the performance evaluation of teachers in universities for TCM, we should further mobilize teachers' participation enthusiasm. The motivation of teachers should not only pay attention to the reward and punishment function of performance evaluation, but also the development function of performance evaluation. In addition, the universities for TCM should analyze the performance evaluation results, continuously improve the evaluation plan according to the practical operation situation. From the perspective of teacher development, reasonable and effective performance evaluation system can help teachers preferably understand their own work situation, improve their own comprehensive quality of scientific research.From the perspective of school development, through reasonable and effective performance evaluation system to understand the work situation of teachers, find out the potential problems in the teachers' team in order to determine the development strategy of the universities for TCM and enhance their core competitiveness. 


\section{Acknowledgement}

In this paper, the research was sponsored by scientific research project of Education Department of Hubei (Project No. Q 20172010)

Ting Kong and Chunyan Li contributed equally to this work. Yuan Sun is the corresponding author.

\section{References}

[1] YU Hong-jie, JIAO Li-xin, DOU Peng. Scientific Research Performance Evaluating Model Based on the Treatment of AHPs Data[J]. Jounal of Anhui Science and Technology University, 2008 (22) 28-23.

[2] ZHANG Yaotian, JIA M ingshun, ZHANG Xucheng, The Evaluation of University research Performance Based on Adaptive Analytic Hierarchy Process[J]. Science and Technology Management Research, 2016.106-11.

[3] WANG Liang, Study on Performance Evaluation System of university Teacher[D]. Qingdao, Qingdao University, 2008.

[4] Qiao Kaiwen, The Evaluation System of Scientific Research Performance in Reseach-oriented Universities[D]. Lanzhou, Lanzhou University, 2009. 